\title{
Direct numerical simulation of developed compressible flow in square ducts
}

\author{
Davide Modesti ${ }^{* a, b}$, Sergio Pirozzoli ${ }^{\mathrm{c}}$, Francesco Grasso ${ }^{\mathrm{a}}$ \\ a Cnam-Laboratoire DynFluid, 151 Boulevard de L'Hopital, 75013, Paris \\ ${ }^{\mathrm{b}}$ Department of Mechanical Engineering, The University of Melbourne, Victoria 3010, Australia \\ ${ }^{\mathrm{c}}$ Dipartimento di Ingegneria Meccanica e Aerospaziale, Sapienza Università di Roma, via Eudossiana 18, Roma, 00184, Italia
}

\section{A R T I C L E I N F O}

\section{Keywords:}

Duct flow

Compressible flows

Wall turbulence

Direct numerical simulation

\begin{abstract}
A B S T R A C T
We carry out direct numerical simulation of compressible square duct flow in the range of bulk Mach numbers $M_{b}=0.2-3$, and up to friction Reynolds number $R e_{\tau}=500$. The effects of flow compressibility on the secondary motions are found to be negligible, with the typical Mach number associated with the cross-stream flow always less than 0.1. As in the incompressible case, we find that the wall law for the mean streamwise velocity applies with good approximation with respect to the nearest wall, upon suitable compressibility transformation. The same conclusion also applies to a passive scalar field, whereas the mean temperature does not exhibit inertial layers because of nonuniformity of the aerodynamic heating. We further find that the same temperature/velocity relation that holds for planar channels is applicable with good approximation for square ducts, and develop a similar relation between temperature and passive scalars.
\end{abstract}

\section{Introduction}

Internal flows in square ducts are common in many engineering applications involving both incompressible and compressible flows. Typical low-speed applications involve cooling, water draining and ventilation systems, whereas at high speed the interest is mainly for aircraft air intakes. Square duct flow exhibits secondary motions in the cross-steam plane. These were first experimentally observed by Nikuradse (1926) and Prandtl (1927), who invoked the occurrence of eight counter-rotating vortices bringing high-momentum fluid from the duct core towards the corners to explain the bending of the streamwise velocity isolines. A considerable number of experiments and numerical simulations have been produced to explain the nature of secondary motions. In particular the present authors have recently developed (Pirozzoli et al., 2018; Modesti and Pirozzoli, 2018), a direct numerical simulation (DNS) dataset of square duct flow in the friction Reynolds number range $R e_{\tau}^{*}=h / \delta_{v}^{*} \approx 150-1000$ (where $h$ is the duct half side length, and $\delta_{v}^{*}=v_{w} / u_{\tau}^{*}$ is the viscous length scale based on the mean friction velocity $u_{\tau}^{*}=\sqrt{\tau_{w}^{*} / \rho_{w}}$ ), the highest currently available in the literature. Despite their effect in redistributing the wall shear stress along the duct perimeter, we have shown that secondary motions do not have large influence on the bulk flow properties, and the streamwise velocity field can be characterized with good accuracy as resulting from the superposition of four flat walls in isolation. Furthermore, we showed that secondary motions contribute approximately $6 \%$ of the total friction, and act as a self-regulating mechanism of turbulence whereby wall shear stress non-uniformities induced by corners are equalized, and universality of the wall-normal velocity profiles is established.

Regarding the compressible flow regime experimental and numerical studies are rather limited. Davis et al. (1986) investigated supersonic developing adiabatic flow at inlet Mach number $M=3.91$ and unit Reynolds number $R e / \mathrm{m}=1.8 \times 10^{6}$. They found that secondary motions develop as in the incompressible case and that the transformed van Driest velocity profiles obey the universal logarithmic wall law. More recently, Morajkar and Gamba (2016) carried out a series of experiments for supersonic duct flow at $M=2.75, R e / \mathrm{m}=8.9 \times 10^{6} \mathrm{using}$ stereoscopic particle image velocimetry. Similar to the incompressible case, they found that the velocity isolines bulge towards the duct corners due to eight counter-rotating cross-stream vortices. The prediction of secondary motions is notoriously difficult for turbulence models, especially for those based on the scalar eddy-viscosity hypothesis (Bradshaw, 1987). Mani et al. (2013) carried out Reynolds averaged Navier-Stokes simulations of supersonic square duct flow using different eddy viscosity models, showing that satisfactory prediction of secondary motions is recovered using quadratic constitutive relations. Vázquez and Métais (2002) performed large-eddy simulation (LES) of compressible isothermal duct flow at bulk Mach number $M_{b}=u_{b} / c_{w}=0.5$ (with $u_{b}$ the bulk velocity and $c_{w}$ the speed of sound at the wall), both with cooled walls and only one heated wall. The cooled

\footnotetext{
* Corresponding author at: Department of Mechanical Engineering, The University of Melbourne, Victoria 3010, Australia.

E-mail address: davide.modesti@unimelb.edu.au (D. Modesti).
} 
case showed good agreement with incompressible data available in the literature, indicating that compressibility effects are negligible at that Mach number, whereas higher intensity of the secondary motions was observed for the case with one heated wall. Vane and Lele (2015) carried out wall-modeled LES corresponding to the experimental setup of Davis et al. (1986), and found that the development of the secondary eddies is strongly affected by the wall shear stress distribution, and that they can significantly alter the primary, axial flow. This is consistent with the findings of the present authors, who carried out numerical experiments of incompressible duct flow in which secondary motions have been artificially suppressed (Modesti et al., 2018) and found that both the wall shear stress and mean streamwise velocity are affected.

Although available studies of compressible duct flow seem to agree that the structure of the secondary motions is weakly affected by compressibility, the quantitative effect of Mach number variations on the flow is not fully understood yet. In particular, an important practical issue is the definition of the relevant effective Reynolds number for comparison across Mach numbers, which is intrinsically related to the subject of compressibility transformations (Morkovin, 1962). In plane channel flow, Modesti and Pirozzoli (2016) found that the compressibility transformation derived by Trettel and Larsson (2016, hereafter referred to as TL) yields very good collapse of the wall-scaled velocity distributions in a wide range of Mach numbers. On the other hand the applicability and accuracy of compressibility transformations has never been assessed in the case of multiple walls, and success of TL transformation in plane channel flow does not automatically guarantee translate to success in the case of duct flow. Another topic of interest is the behavior of passive scalars in compressible flow, which is important to understand mixing processes in turbulent combustion. However, passive scalars in compressible wall-bounded flows have received little attention so far, mainly limited to the case of planar channels (Foysi and Friedrich, 2005) and pipe flow (Ghosh et al., 2008; 2010). Another important topic in compressible flows is temperature/ velocity relations (Smits and Dussauge, 2006). Whereas these relations are well established in canonical flows (Zhang et al., 2014), their validity has never been verified for more complex geometries.

The aim of the present work is three-fold. First, we attempt to extend the compressibility velocity transformations developed for plane channel flow to the case of multiple walls, through suitable definition of the relevant effective Reynolds number. Second, we propose a compressibility transformation for passive scalars. Third, we analyze the temperature field with the main objective of verifying the validity of temperature/velocity relations. Hence, we perform DNS of isothermalwall square duct flow in the range of bulk Mach number $M_{b}=0.2-3$, up to $R e_{\tau}^{*}=500$.

\section{Computational setup}

We solve the compressible Navier-Stokes equations for a perfect shock-free heat-conducting gas augmented with the transport equation for a passive scalar $\phi$, $\frac{\partial \rho}{\partial t}+\frac{\partial \rho u_{j}}{\partial x_{j}}=0$

$\frac{\partial \rho u_{i}}{\partial t}+\frac{\partial \rho u_{i} u_{j}}{\partial x_{j}}=-\frac{\partial p}{\partial x_{i}}+\frac{\partial \sigma_{i j}}{\partial x_{j}}+\Pi \delta_{i 1}$

$\frac{\partial \rho s}{\partial t}+\frac{\partial \rho u_{j} s}{\partial x_{j}}=\frac{1}{T}\left(-\frac{\partial q_{j}}{\partial x_{j}}+\sigma_{i j} \frac{\partial u_{i}}{\partial x_{j}}\right)$

$\frac{\partial \rho \phi}{\partial t}+\frac{\partial \rho u_{j} \phi}{\partial x_{j}}=\frac{\partial}{\partial x_{j}}\left(\rho \alpha \frac{\partial \phi}{\partial x_{j}}\right)+\Phi$,

where $u_{i}$ is the velocity component in the ith direction, $\rho$ is the fluid density, $p$ is the thermodynamic pressure, $s=c_{v} \ln \left(p \rho^{-\gamma}\right)$ is the entropy per unit mass, $\gamma=c_{p} / c_{v}=7 / 5$ is the specific heat ratio, $\sigma_{i j}$ and $q_{j}$ are, respectively, the viscous stress and the heat flux components,

$\sigma_{i j}=\mu\left(\frac{\partial u_{i}}{\partial x_{j}}+\frac{\partial u_{j}}{\partial x_{i}}-\frac{2}{3} \frac{\partial u_{k}}{\partial x_{k}} \delta_{i j}\right)$,

$q_{j}=-k \frac{\partial T}{\partial x_{j}}$.

The dependence of the viscosity coefficient on temperature is accounted for through Sutherland's law, and the thermal conductivity is defined as $k=c_{p} \mu / P r$, with $P r=0.71$. The unsteady, uniform-in-space forcing term $\Pi$ in Eq. (1b)is evaluated at each time step in order to discretely enforce constant mass-flow-rate in time, hence the bulk Mach number is also constant. The passive scalar diffusivity is $\alpha=\mu / \rho S c$, with the Schmidt number, and the forcing term $\Phi$ in Eq. (1d) is evaluated at each time step to keep a constant scalar flow rate in time. The equations are numerically solved using a fourth-order co-located finite-difference solver, and the convective terms are discretized in such a way that the total kinetic energy is preserved from convection in the inviscid limit (Pirozzoli, 2010). Viscous terms are expanded to Laplacian form and discretized using standard central finite-difference approximations. The use of the entropy equation (1c) in place of the total energy equation is instrumental to the semi-implicit time advancement, thus avoiding the severe acoustic time step limitation in the wall-normal direction (Modesti and Pirozzoli, 2018). The equations are solved in a box of size $6 \pi h \times 2 h \times 2 h$, which was found to yield satisfactory insensitivity of the flow statistics (Pirozzoli et al., 2018). Periodicity is enforced in the streamwise direction, whereas isothermal no-slip boundary conditions are used at the walls, where we also set $\phi=0$ (Modesti and Pirozzoli, 2016). The velocity field is initialized with the incompressible laminar solution with superposed synthetic perturbations obtained through the digital filtering technique (Klein et al., 2003). Density and temperature are initially uniform, whereas the passive scalar is initialized as the streamwise velocity field, upon suitable rescaling. Streamwise- and time-averaged statistics have been collected at equal time intervals, and convergence of the flow statistics has been checked a-posteriori. As observed in previous DNS studies of duct flow (Pinelli et al., 2010; Vinuesa et al.,

Table 1

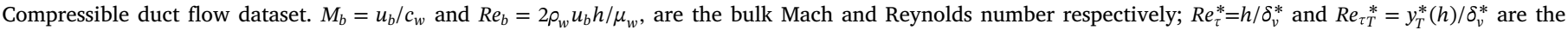

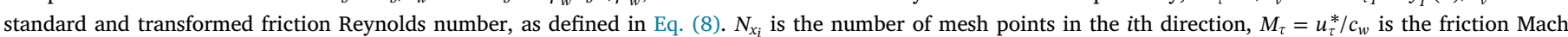

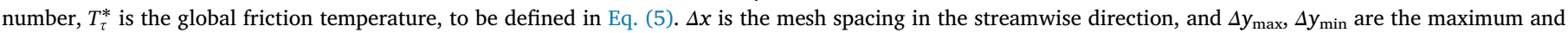

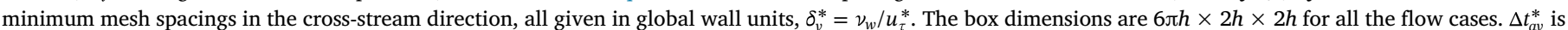
the effective time averaging interval.

\begin{tabular}{|c|c|c|c|c|c|c|c|c|c|c|c|c|c|c|}
\hline Case & $M_{b}$ & $R e_{b}$ & $R e_{\tau}^{*}$ & $R e_{\tau T}^{*}$ & $M_{c}$ & $N_{x}$ & $N_{y}$ & $N_{z}$ & $\Delta x^{*}$ & $\Delta y_{\max }^{*}$ & $\Delta y_{\min }^{*}$ & $M_{\tau}$ & $T_{\tau}^{*} / T_{w}$ & $\Delta t_{a v}^{*} u_{\tau}^{*} / h$ \\
\hline D02 & 0.2 & 4410 & 152 & 146 & 0.26 & 512 & 128 & 128 & 5.6 & 3 & 0.68 & 0.014 & 0.001 & 2290 \\
\hline D15A & 1.5 & 6000 & 228 & 141 & 1.66 & 512 & 128 & 128 & 8.4 & 5.4 & 0.58 & 0.082 & 0.05 & 830 \\
\hline D15B & 1.5 & 14600 & 507 & 332 & 1.62 & 1024 & 256 & 256 & 9.3 & 6.0 & 0.76 & 0.075 & 0.045 & 1036 \\
\hline D3 & 3 & 9760 & 483 & 145 & 2.42 & 1024 & 256 & 256 & 8.9 & 5.8 & 0.61 & 0.12 & 0.14 & 213 \\
\hline
\end{tabular}


(a)

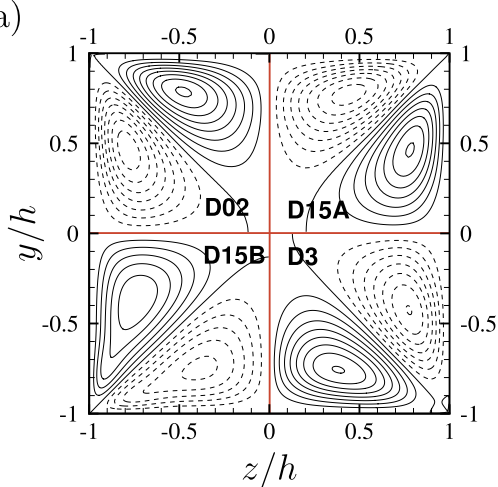

(b)

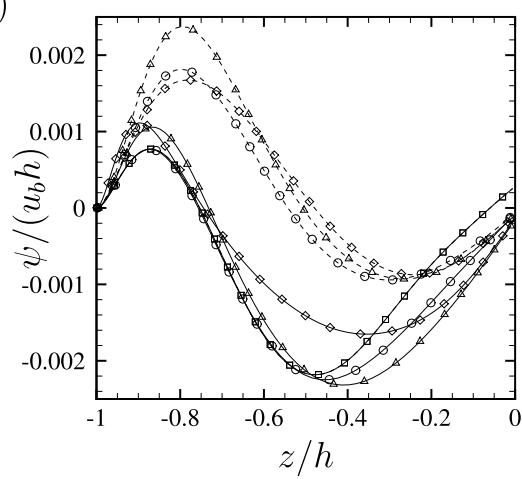

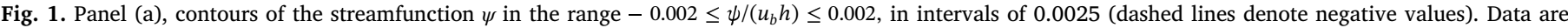

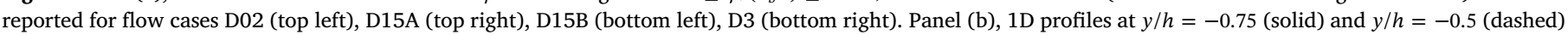
for case D02 (deltas), D15A (circles), D15B (diamond), D3 (squares).

(a)

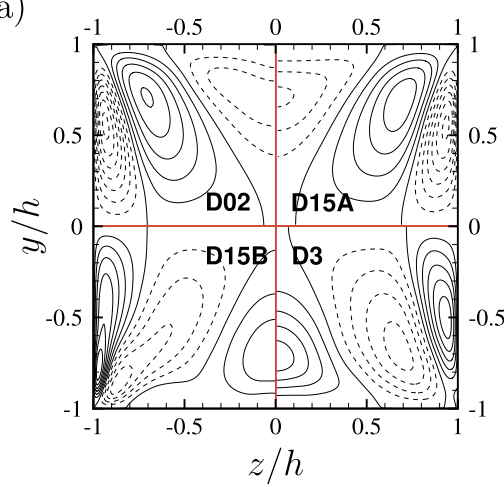

(b)

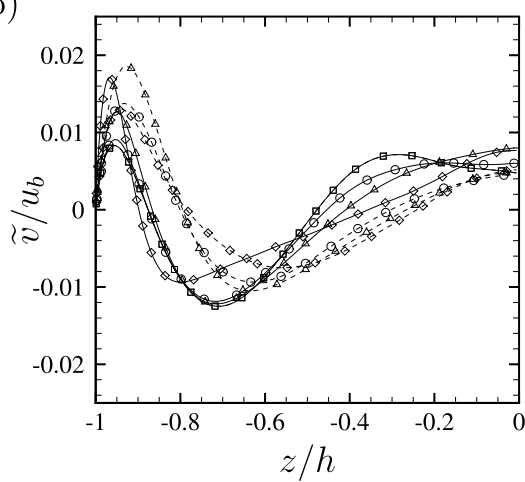

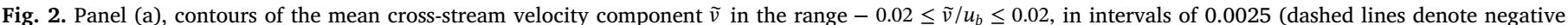

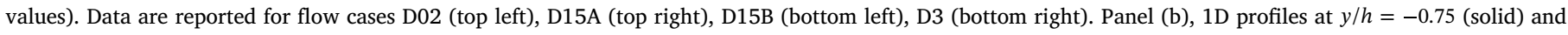
$y / h=-0.5$ (dashed) for case D02 (deltas), D15A (circles), D15B (diamond), D3 (squares).

2016; Pirozzoli et al., 2018), the time integration intervals needed to achieve statistical convergence are much longer than those typical of plane channel flow; see Table 1. Duct flow statistics inevitably feature asymmetries in the cross-stream plane, even after long time averaging (Pirozzoli et al., 2018), which are here alleviated through averaging over the four quadrants. Three supersonic simulations have been carried out at $M_{b}=1.5, R e_{\tau}^{*}=220-500$ and at $M_{b}=3, R e_{\tau}^{*}=500$, (see Table 1). A reference low-speed simulation at $M_{b}=0.2, R e_{\tau}^{*}=150$ has also been carried out (case D02), which was shown to yield excellent agreement of mean and r.m.s. velocity with reference incompressible DNS data (Pirozzoli et al., 2018).

The maximum Mach number $M_{c}=\tilde{u}_{c} / c$ are also reported. We note that $M_{c}<M_{b}$ for flow case D3, due to the fact that the bulk density is constant for all flow cases and $M_{b}=u_{b} / \rho_{b}, u_{b}=1 / \rho_{b} \int_{V} \rho u \mathrm{~d} V$, where $V$ is the flow volume.

For the forthcoming analysis, the results are reported both in local and global wall units. Accordingly, we introduce reference friction values for velocity, temperature, and passive scalar, namely

$u_{\tau}^{2}=\left.v_{w} \frac{\partial \tilde{u}}{\partial y}\right|_{w}, \quad u_{\tau}^{* 2}=\frac{h \bar{\Pi}}{2 \rho_{w}^{*}}$,

$T_{\tau}=\left.\frac{k_{w}}{\rho_{w} c_{p} u_{\tau}} \frac{\partial \widetilde{T}}{\partial y}\right|_{w}, \quad T_{\tau^{*}}=\frac{h \bar{\Psi}}{2 \rho_{w}^{*} c_{p} u_{\tau^{*}}}$,

$\phi_{\tau}=\left.\frac{\alpha_{w}}{u_{\tau}} \frac{\partial \widetilde{\phi}}{\partial y}\right|_{w}, \quad \phi_{\tau^{*}}=\frac{h \bar{\Phi}}{2 \rho_{w}^{*} u_{\tau^{*}}}$

where the $w$ subscript denotes wall values, the * superscript quantities averaged over the duct perimeter, and $\bar{\Phi}$ is the viscous dissipation function, defined in Eq. (12). For clarity of notation, hereafter $x$ denotes the streamwise direction, and $y$ and $z$ the cross-stream and wall-normal directions, and $u, v$ and $w$ are the respective velocity components. Both Reynolds $\left(\phi=\bar{\phi}+\phi^{\prime}\right)$ and Favre $\left(\phi=\widetilde{\phi}+\phi^{\prime \prime}, \widetilde{\phi}=\overline{\rho \phi} / \bar{\rho}\right)$ decompositions will be considered in the following, where the overline symbol denotes averaging in the streamwise directions and in time. Accordingly, the Reynolds stress components are denoted as $\tau_{i j}=\bar{\rho} \widetilde{u_{i}^{\prime \prime} u_{j}^{\prime \prime}}$.

\section{Results}

\subsection{Velocity field}

In this section we analyze the structure of the mean velocity field including the secondary motions, with special reference to establishing the effect of compressibility on the validity of compressibility transformations for the wall law. The structure of the secondary motions is hereafter analyzed by introducing a cross-flow stream function $\psi$, defined such that at any point over the duct cross section

$-\bar{\rho} \widetilde{v}=\bar{\rho}_{w} \frac{\partial \psi}{\partial z}, \quad \bar{\rho} \widetilde{w}=\bar{\rho}_{w} \frac{\partial \psi}{\partial y}$,

which satisfies mass conservation in the cross-stream plane. In Fig. 1 we show $\psi$ in a quarter of the duct, scaled with respect to $u_{b}$ and $h$, for the various flow cases of Table 1 . All the flow cases exhibit the same typical flow topology with eight counter-rotating eddies, which act to feed the low-momentum regions created at the corners. Fig. 2 further shows that the cross-stream velocity component, $v(y, z)=-w(z, y)$, is characterized by a three-lobe structure, as in the incompressible case (Pirozzoli et al., 2018). The cross-stream velocity peaks are found 
(a)

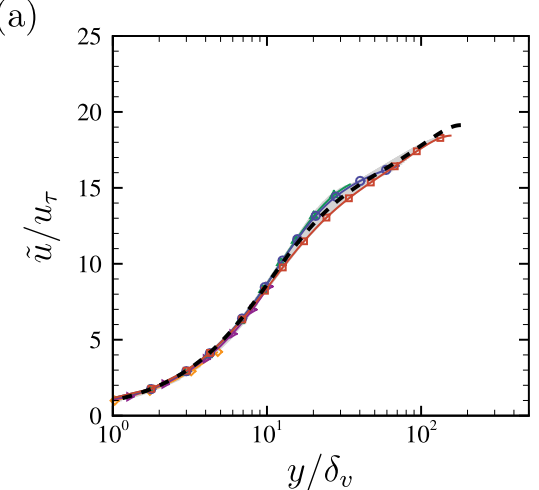

(c)

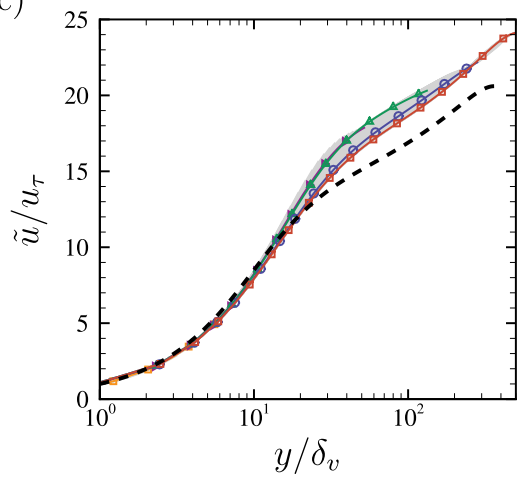

(b)

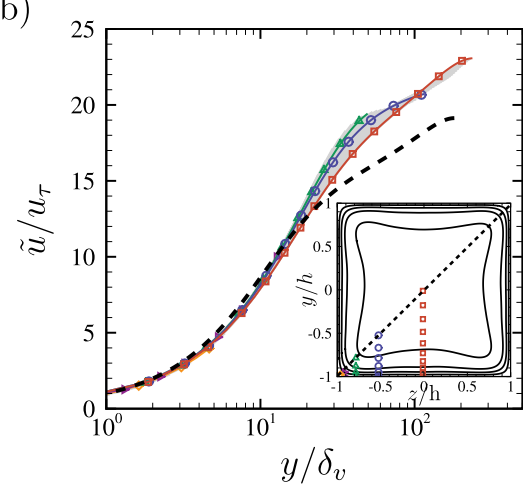

(d)

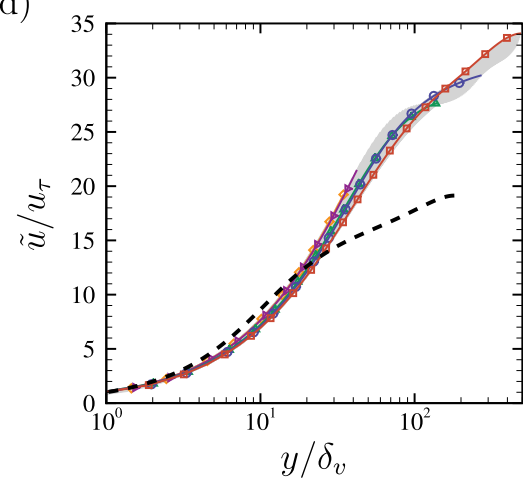

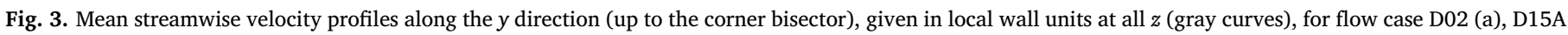

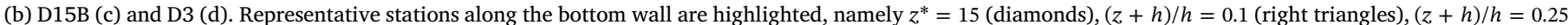

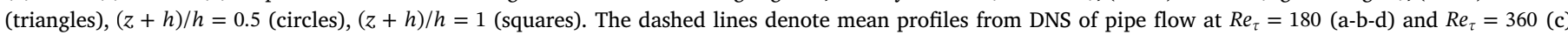
from El Khoury et al. (2013), The inset panel (b) shows the mean streamwise velocity in the cross-stream plane with symbols denoting representative sections.

(a)

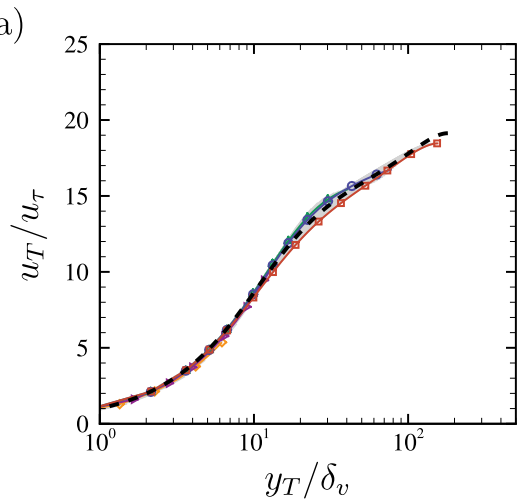

(c)

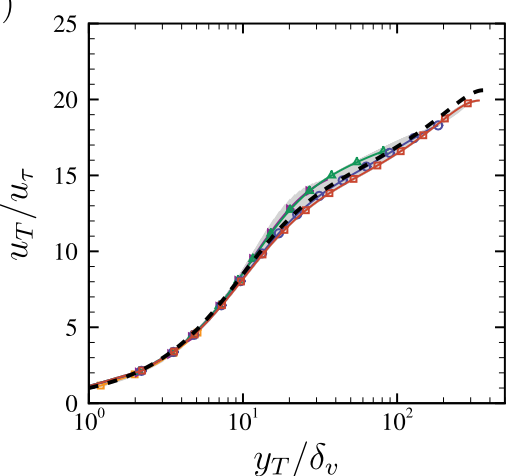

(b)

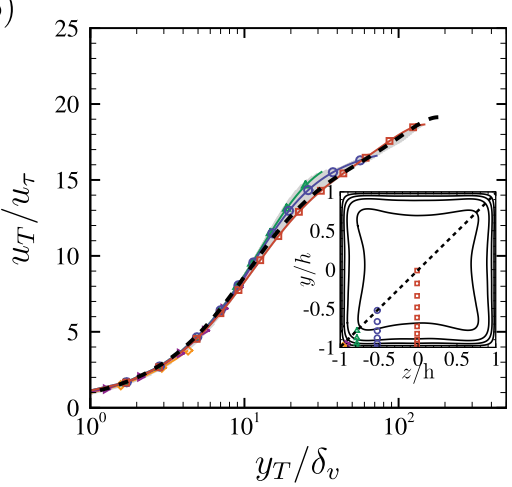

(d)

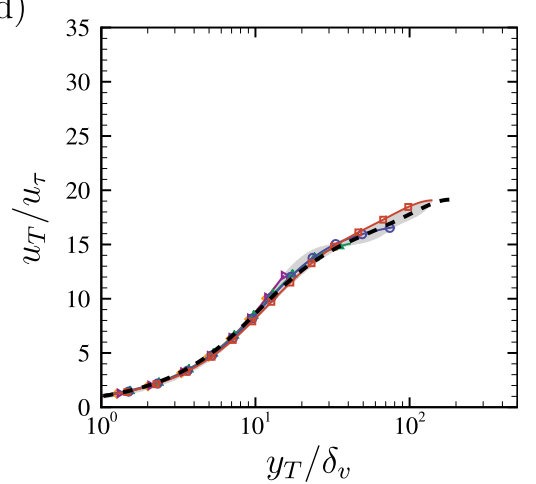

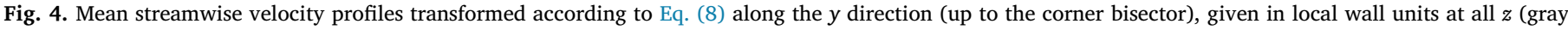

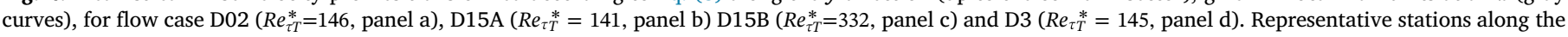

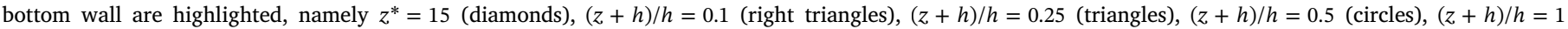

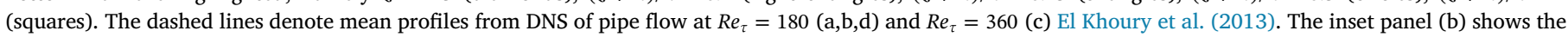
mean streamwise velocity in the cross-stream plane with symbols denoting representative sections. 
(a)

(c)
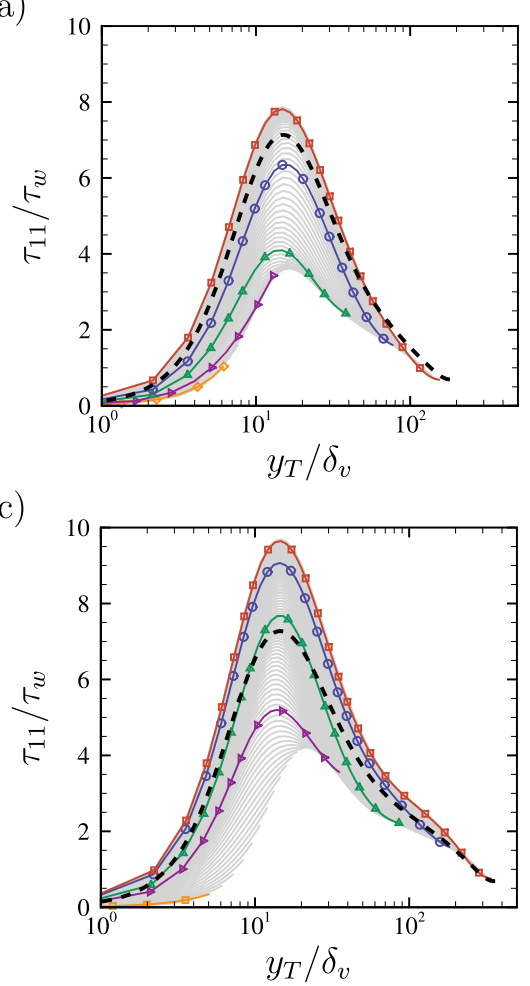

(b)

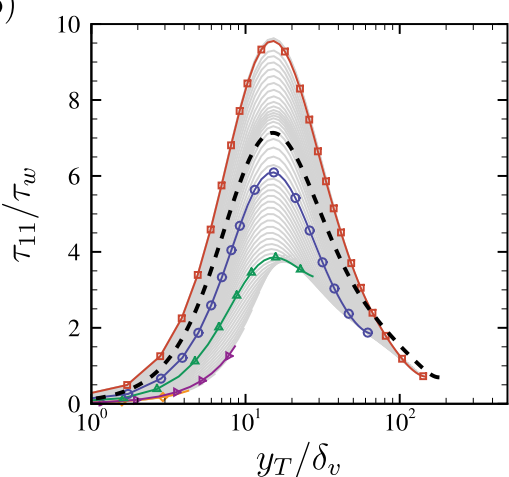

(d)

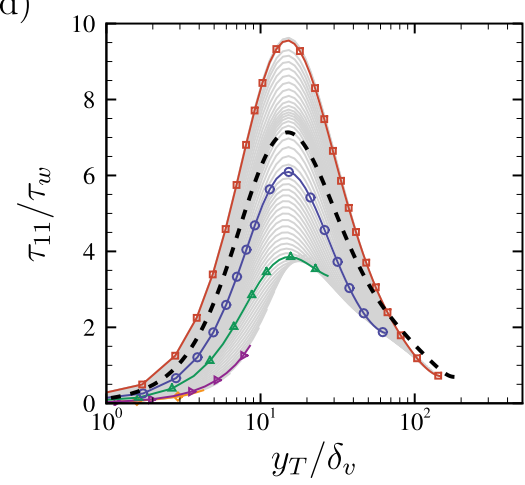

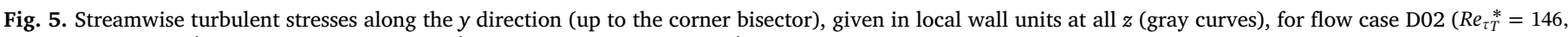

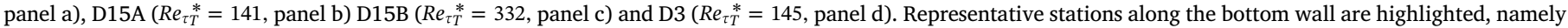

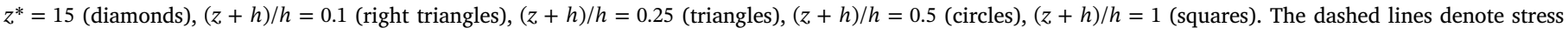
profiles from DNS of pipe flow at $R e_{\tau}=180$ (a,b,d) and $R e_{\tau}=360$ (c) (El Khoury et al., 2013).

to scale reasonably well with the bulk flow velocity, regardless of Mach and Reynolds number, with maximum value of about $2 \%$ of $u_{b}$, which is similar to the incompressible case (Pirozzoli et al., 2018).

The mean streamwise velocity field in compressible flows is generally characterized using compressibility transformations. Morkovin (1962) first postulated that if density fluctuations are negligible with respect to local mean density, the direct effect of compressibility on turbulence reduces to variations of the mean thermodynamic properties. This led to the well known van Driest transformation (van Driest, 1951), which is quite accurate for adiabatic walls, whereas it is known to fail for isothermal walls (Modesti and Pirozzoli, 2016). For the latter wall conditions, Trettel and Larsson (2016) have recently derived a compressibility transformation for channel flow which relies on the mean momentum balance and log-law universality. Pirozzoli et al. (2018) showed that in incompressible square duct flow the streamwise velocity field is mainly influenced by the nearest wall, thus the wall law applies with reasonable accuracy up to the corner bisector. Based on this result and on the fact that the secondary motions are not greatly affected by compressibility, we then introduce the TL transformation for $y$ (the direction normal to the nearest wall) and $u$,

$y_{T}(y, z)=\int_{0}^{y} f_{T}(\eta, z) \mathrm{d} \eta, \quad u_{T}(y, z)=\int_{0}^{y} g_{T}(\eta, z) \frac{\partial \tilde{u}}{\partial \eta}(\eta, z) \mathrm{d} \eta$,

where the stretching functions are defined as

$f_{T}(y, z)=\frac{\partial}{\partial y}\left(\frac{y}{R^{1 / 2} N}\right), \quad g_{T}(y, z)=R N \frac{\partial}{\partial y}\left(\frac{y}{R^{1 / 2} N}\right)$,

with $N(y, z)=\bar{\nu} / \bar{\nu}_{w}$, and $R(y, z)=\bar{\rho} / \bar{\rho}_{w}$. It is important to note that the stretching functions $f_{T}$ and $g_{T}$ depend both on $y$ and $z$ (through the mean density and mean viscosity), and likewise $y_{T}$ and $u_{T}$. It is also important to note that the transformation used for the wall distance is actually equivalent to use of the semi-local wall units introduced by Huang et al. (1995).
Fig. 3 shows the mean velocity profiles as a function of the wallnormal distance up to the corner bisector (where mean velocity attains a maximum), in local wall units. For reference purposes, the mean velocity profiles from DNS of incompressible pipe flow are also reported (El Khoury et al., 2013). Reasonable collapse of the velocity profiles at various $z$ is recovered, also including the near-corner region. However, large differences are found among the different flow cases, especially at higher Mach number. The TL transformed velocity profiles are shown in Fig. 4, which now shows much better collapse of the velocity distributions both with respect to $z$ (up to the corner bisector) and across different flow cases. This confirms on one hand that the TL transformation derived for plane channel flow also holds with good approximation for square ducts. On the other hand, the figure also suggests that for the Mach numbers considered here close similarity with the velocity distributions in incompressible pipe flow is recovered for matching values of an equivalent friction Reynolds number, which we define as

$R e_{\tau T}^{*}=y_{T}^{*}(h) / \delta_{v}^{*}$.

where

$y_{T}^{*}(y)=\frac{1}{h} \int_{0}^{h} y_{T}(y, z) \mathrm{d} z$,

is a stretched wall-normal coordinate averaged along the $z$ direction, which accounts in the mean for the variation of the local transformed scale $y_{T}$ with $z$. As in the incompressible case, duct flow shows close similarity with pipe flow, which is a direct consequence of the fact that the intensity of the secondary flows is rather small (Pirozzoli et al., 2018).

The streamwise turbulent stress $\left(\tau_{11}\right)$ normalized by the local wall shear stress is shown in Fig. 5, and compared with incompressible pipe flow data. Along most of the wall, the behaviour is qualitatively similar to canonical pipe flow, with a near-wall peak at $y^{+} \approx 12$. The scatter 
(a)

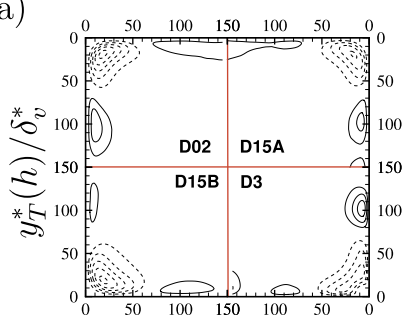

(c)

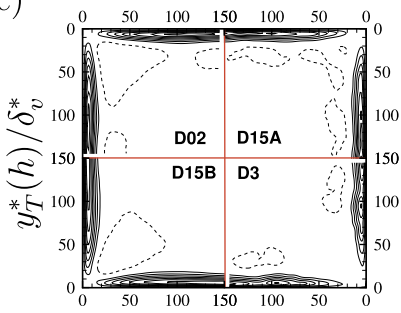

(e)

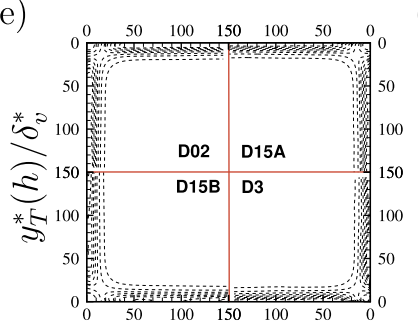

$(\mathrm{g})$

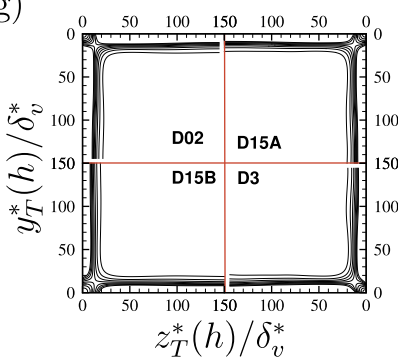

(b)

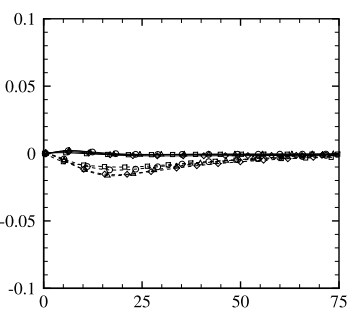

(d)

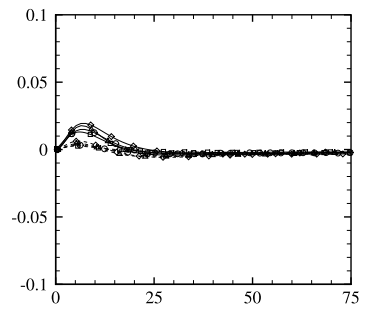

(f)

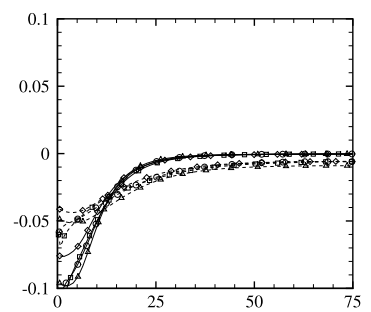

(h)

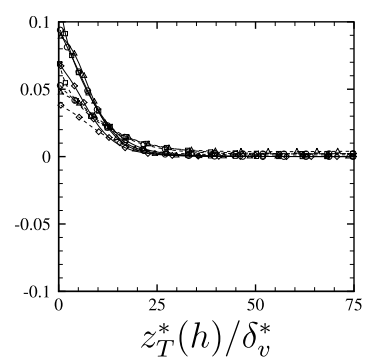

Fig. 6. Contours (panels a, c, e, g), and profiles (panels b, d, f, h) of mean enthalpy budget terms (Eq. (12)) in global stretched inner units. Contours are shown in the range $-0.025 \leq() /.\left(\rho_{w}^{*} u_{\tau}^{*} T_{\tau}^{*} / \delta_{v}^{*}\right) \leq 0.025$, in intervals of $3 \cdot 10^{-3}$, dashed lines denoting negative values. From top to bottom we show convection, turbulent transport, viscous diffusion, and viscous dissipation. The 1D profiles are reported at $y^{*}=25$ (dashed lines) and $y^{*}=75$ (solid lines), for all cases (D02, triangles; D15A, circles; D15B, diamonds; D3, squares).

among the various $z$ sections appears to be generally much larger than for the mean velocity field, although it seems to become confined to the corner vicinity at high enough $R e$. The streamwise turbulent stress component exhibits a higher peak in the buffer layer at supersonic Mach number, which is not accurately captured by normalization with the local wall shear stress. This effect was observed in previous studies of canonical compressible flows (Coleman et al., 1995; Ghosh et al., 2010; Modesti and Pirozzoli, 2016), but no convincing explanation has been provided to date. Good collapse with incompressible pipe flow is by the way observed for all cases in the outer part of the wall layer. All other Reynolds stress components (not shown) have similar or higher degree of universality.

\subsection{Temperature field}

The temperature field is herein analyzed starting from the averaged enthalpy transport equation,

$$
\begin{aligned}
\underbrace{\frac{\partial \bar{\rho} c_{p} \tilde{v} \widetilde{T}}{\partial y}+\frac{\partial \bar{\rho} c_{p} \widetilde{w} \widetilde{T}}{\partial z}}_{C} & =\underbrace{\frac{\partial}{\partial y}\left(\bar{k} \frac{\partial \widetilde{T}}{\partial y}\right)+\frac{\partial}{\partial z}\left(\bar{k} \frac{\partial \widetilde{T}}{\partial z}\right)}_{D}+ \\
& \underbrace{+\overline{u \frac{\partial p}{\partial x}}+\overline{v \frac{\partial p}{\partial y}}+\overline{w \frac{\partial p}{\partial z}}}_{P}+\underbrace{\overline{\sigma_{i j} \frac{\partial u_{i}}{\partial x_{j}}}}_{\Psi} \underbrace{-\frac{\partial \bar{\rho} c_{p} \widehat{v^{\prime \prime} T^{\prime \prime}}}{\partial y}-\frac{\partial \bar{\rho} c_{p} \overline{w^{\prime \prime} T^{\prime \prime}}}{\partial z}}_{T},
\end{aligned}
$$

where the terms $C, D, P, \Psi$, and $T$ represent convection, viscous diffusion, pressure work, viscous dissipation and turbulent transport. The various contributions to the budget are reported in Fig. 6, in global stretched inner units, upon normalization of temperature with respect to the global friction temperature, both in the cross-stream plane and at selected wall-normal sections. The pressure work term is not reported, being negligible in all cases (Bradshaw and Huang, 1995). The figure shows that the stretched inner scaling yields good collapse across different Mach (cases D02, D15A, D3) and Reynolds numbers (D15A, D15B). Similar to what was found from the mean momentum balance equation in the incompressible case (Pirozzoli et al., 2018), we note that mean convection is mainly relevant at the duct corners, whereas it plays a minor role with respect to the other terms in the core region. Viscous diffusion and dissipation contribute most to the budget, and they are partially balanced by turbulent heat transport in the buffer layer.

Fig. 7 shows the inner-scaled wall-normal temperature profiles up to the corner bisector, highlighting good agreement between duct flow and supersonic pipe flow data at matching Mach and Reynolds number (Modesti, 2017). The temperature profiles show rather good universality with respect to the spanwise direction in the core region, whereas larger scatter is observed between $10<y / \delta_{v}<100$, compared to the mean velocity profiles, Figs. $3-4$. Nevertheless, temperature does not exhibit any logarithmic layer nor universality with respect to Reynolds and Mach number, unlike previously observed for the mean velocity field (see Fig. 4). Corner effects in the temperature field seem to be more significant than for the mean velocity, yielding earlier deviation from a common distribution when approaching the wall. This behavior may be explained by noting that in the current case of isothermal wall the energy balance is mainly controlled by aerodynamic heating, which is associated with viscous dissipation, and which acts as a non-uniform spatial forcing. As shown in the forthcoming Section, in the case of a passive scalar with spatially uniform forcing a logarithmic layer does in fact emerge as for the mean velocity. Hence, deviations of the temperature distributions from a logarithmic behavior are the likely consequence of non-uniform heating, which implies that regarding the temperature as a passive scalar may lead to incorrect conclusions, even at low Mach numbers. It is noteworthy that greater universality of the temperature profiles is achieved in the core region when the temperature profiles are scaled with the average friction temperature $T_{\tau}^{*}$ defined in Eq. (5) (see Fig. 8), which is a hint that the temperature field away from walls is primarily controlled by the overall aerodynamic heating, whereas local heating is important near walls.

Knowledge of the temperature distribution in compressible flow is necessary for the prediction of friction (Smits and Dussauge, 2006). In particular, based on the distribution of $T$ one can derive the mean velocity distribution through reverse application of the compressibility transformation introduced in Section 3.1, for which temperature/velocity relationships are needed. The classical temperature/velocity relation by Walz (1959) has proven its accuracy in the case of adiabatic walls (Duan et al., 2010), whereas it is found to fail in the case of isothermal walls (Modesti and Pirozzoli, 2016). Recently, Zhang et al. (2014) derived the following generalized temperature/ velocity relation,

$\frac{\widetilde{T}}{T_{w}}=1+\frac{T_{r g}-T_{w}}{T_{w}} \frac{\widetilde{u}}{\widetilde{u}_{e}}+\frac{\widetilde{T_{e}}-T_{r g}}{T_{w}}\left(\frac{\widetilde{u}}{\widetilde{u}_{e}}\right)^{2}$,

where $T_{r g}=\tilde{T}_{e}+r_{g} \tilde{u}_{e}^{2} /\left(2 c_{p}\right)$ is a generalized recovery temperature, 

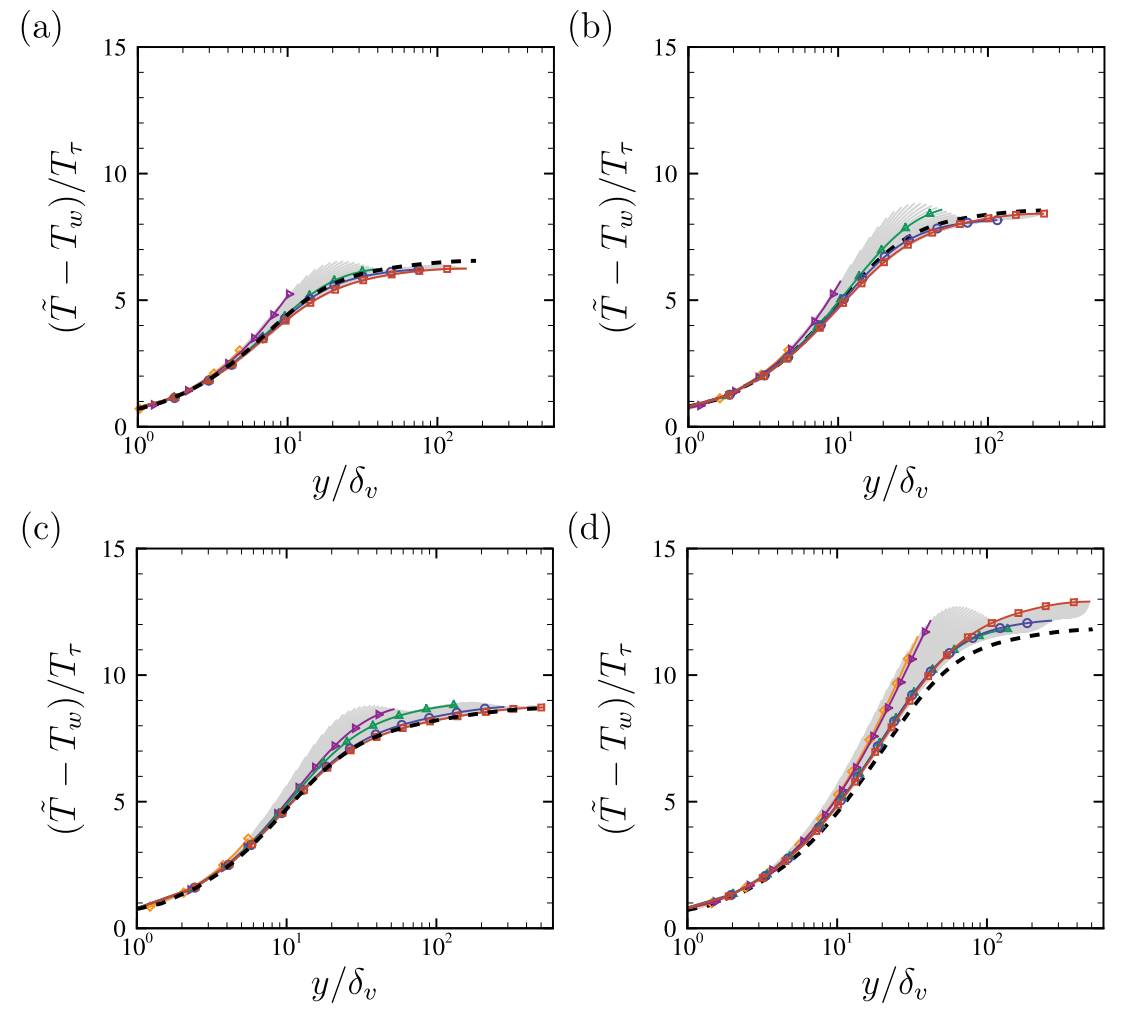

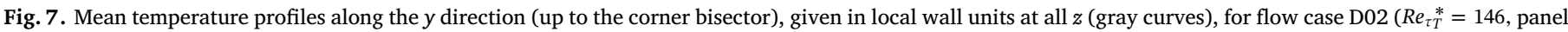

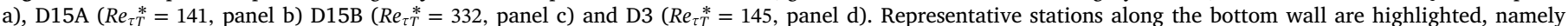

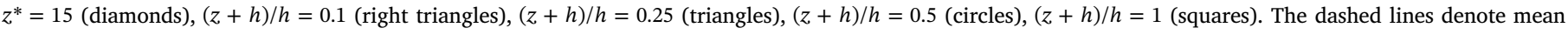
profiles from DNS of pipe flow at $R e_{\tau}=143$ (a,b,d) and $R e_{\tau}=334$ (c) Modesti (2017).
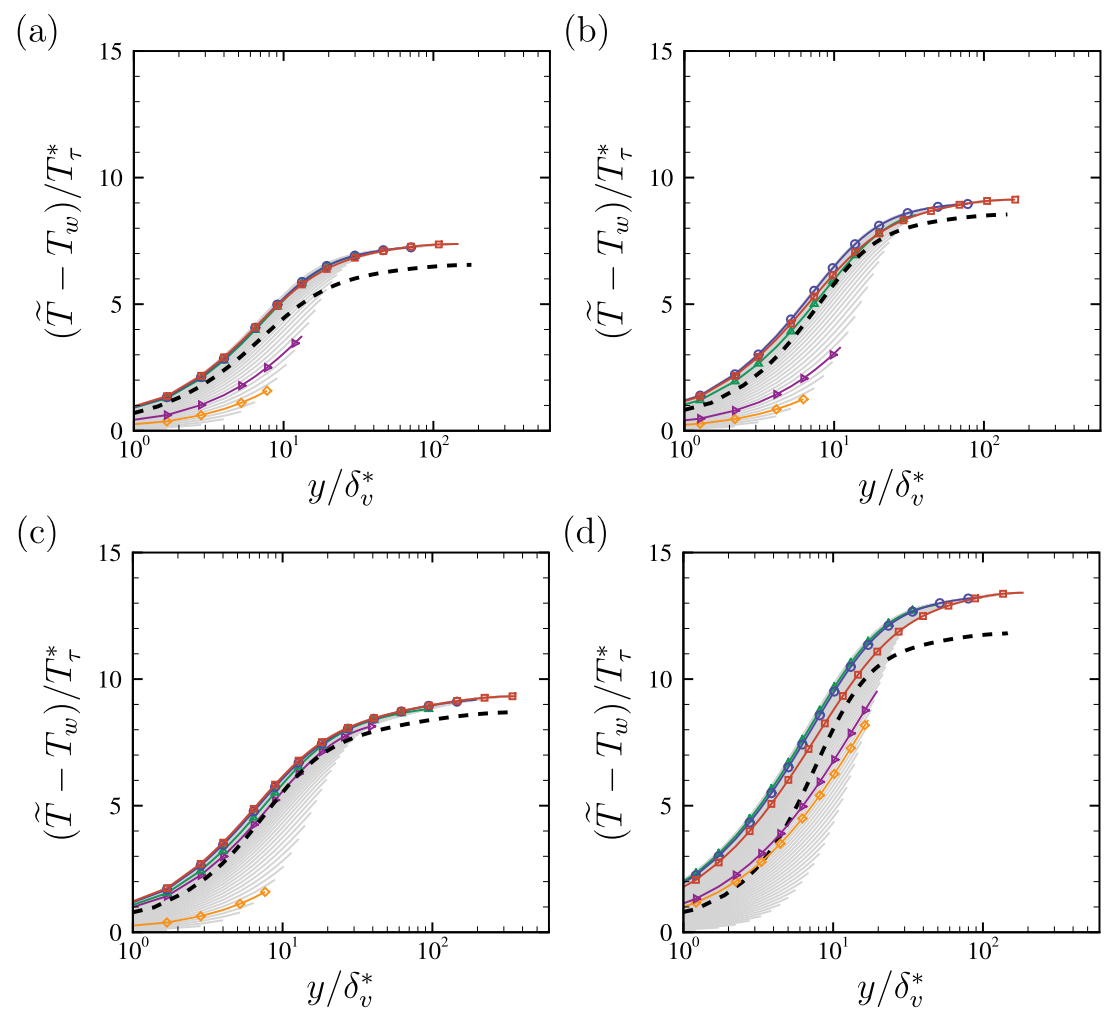

Fig. 8. Mean temperature profiles along the $y$ direction (up to the corner bisector), given in global wall units at all $z$ (gray curves), for flow case D02 (Re $e_{T T}^{*}=146$, panel a), D15A $\left(R e_{\tau T}^{*}=141\right.$, panel b) D15B $\left(R e_{\tau T}^{*}=332\right.$, panel c) and D3 $\left(R e_{\tau T}^{*}=145\right.$, panel d). Representative stations along the bottom wall are highlighted, namely $z^{*}=15$ (diamonds), $(z+h) / h=0.1$ (right triangles), $(z+h) / h=0.25$ (triangles), $(z+h) / h=0.5$ (circles), $(z+h) / h=1$ (squares). The dashed lines denote mean profiles from DNS of pipe flow at $R e_{\tau}=143$ (a,b,d) and $R e_{\tau}=334$ (c) Modesti (2017). 


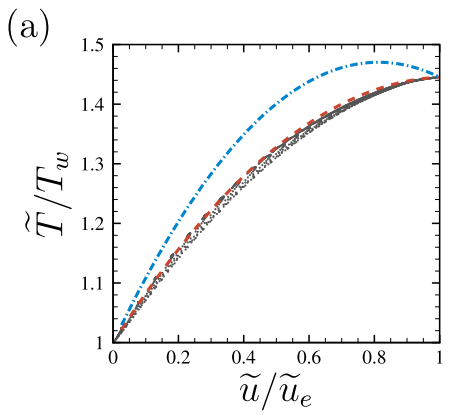

(b)

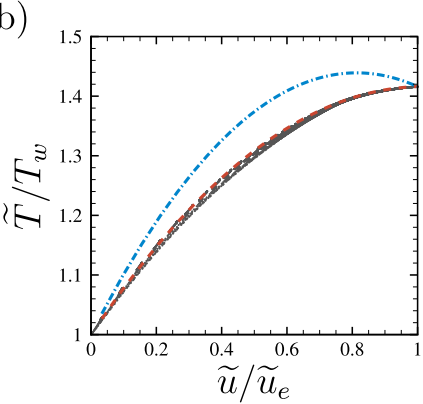

(c)

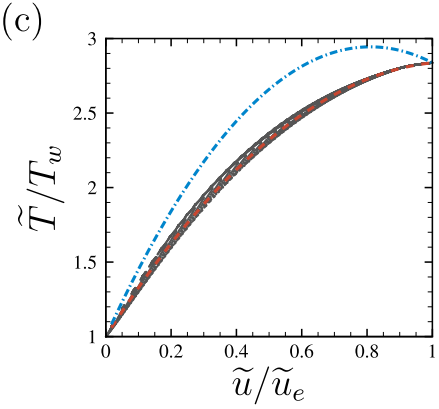

Fig. 9. Scatter plots of mean temperature versus mean velocity for all points in the duct cross section for flow cases D15A (a), D15B (b), D3 (c). The subscript $e$ refers to duct centerline values. The dashed line denotes the generalized temperature/velocity relation of (Zhang et al., 2014) given in Eq. (13) and the dash-dotted line denote Walz relation (Walz, 1959).

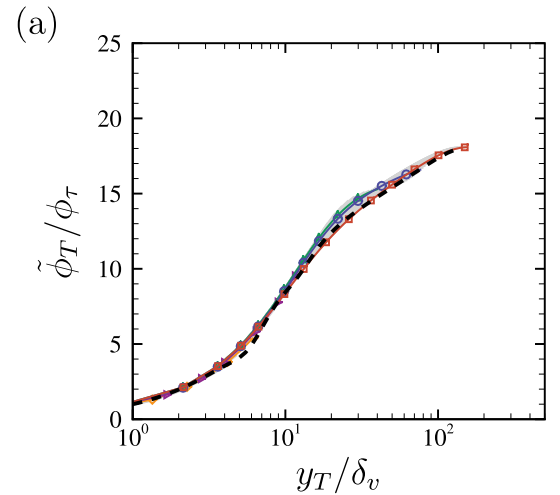

(c)

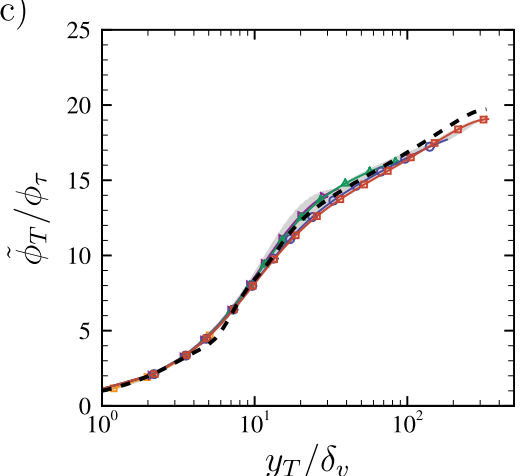

(b)

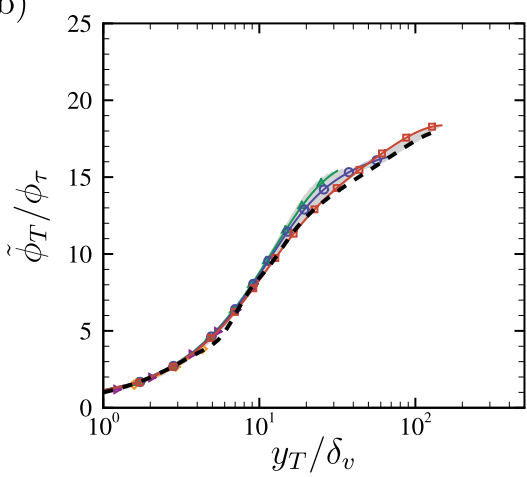

(d)

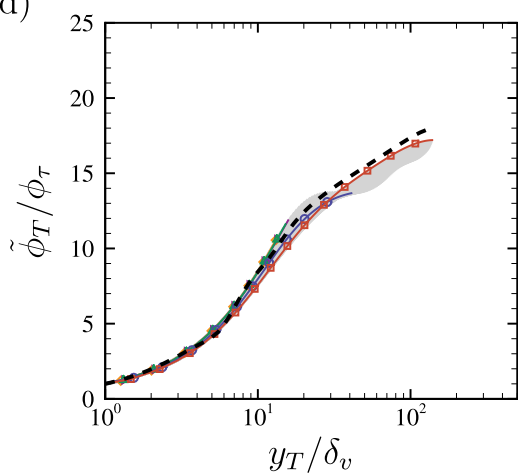

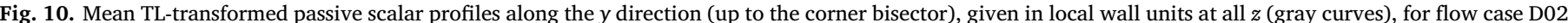

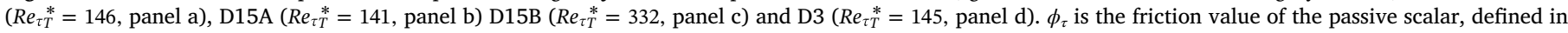

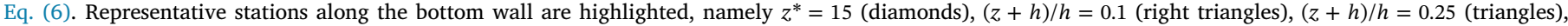

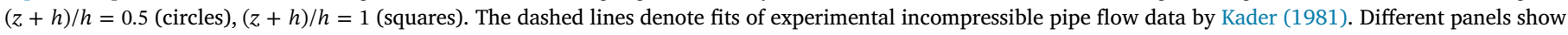
flow cases D02 (a), D15A (b), D15B (c), D3 (d).

(a)

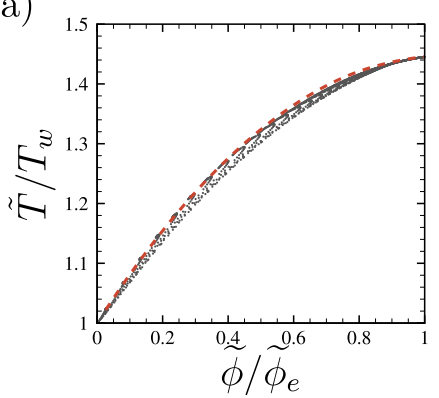

(b)

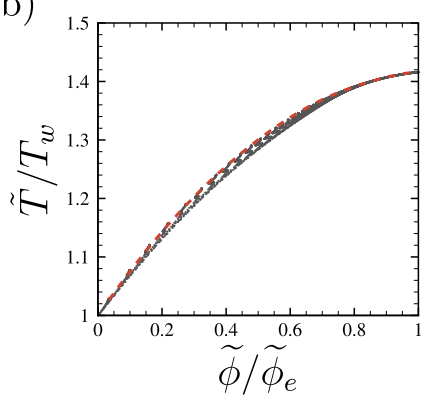

(c)

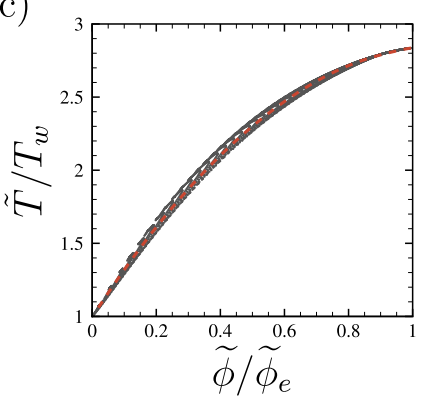

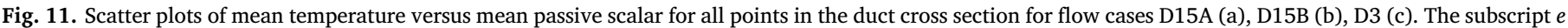
refers to duct centerline values. The dashed line denotes the generalized temperature/passive scalar relation given in Eq. (15).

$r_{g}=2 c_{p}\left(T_{w}-\tilde{T}_{e}\right) / \tilde{u}_{e}^{2}-2 \operatorname{Prq} q_{w} /\left(\tilde{u}_{e} \tau_{w}\right)$ is a generalized recovery factor, and $u_{e}$ and $T_{e}$ are the external values of velocity and temperature, here interpreted as the duct centerline values. Eq. (13) explicitly takes into account the wall heat flux $q_{w}$, and it reduces to the Walz relation in the case of adiabatic walls. Fig. 9 shows scatter plots of temperature as a function of velocity for all points in the duct cross section. Nearly perfect coincidence of the present DNS data with the predictions of Eq. (13) is observed. Integration of Eq. (9) thus allows one to 
(a)

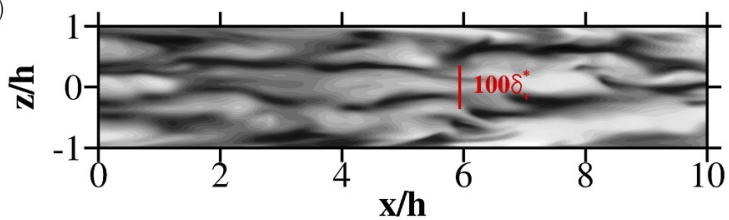

(b)

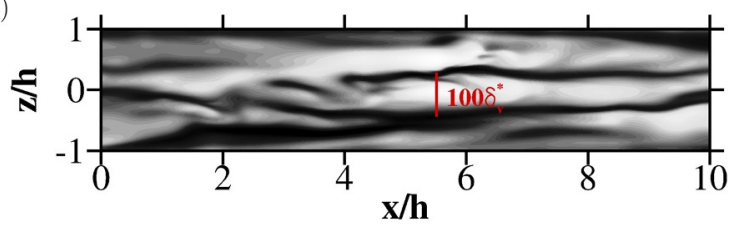

(c)

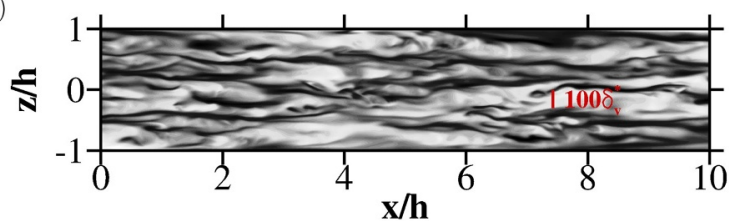

(d)

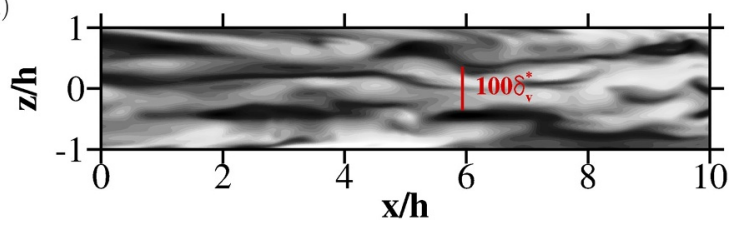

Fig. 12. Instantaneous streamwise velocity fluctuations in wall-parallel plane $\left(y_{T}^{+}=15\right)$, for flow cases D02 (a), D15A (b), D15B (c) and D3 (d). Contours are shown in the range $-3 \leq u^{\prime \prime} / \sqrt{\tau_{11 D}} \leq 3$, from dark to light shades.

reconstruct the full velocity field for given values of the bulk Reynolds and Mach numbers.

\subsection{Passive scalar field}

In this section we study the transport of a passive scalar field governed by Eq. (1d) at unit Schmidt number, previously studied in channel flows in the incompressible case (Pirozzoli et al., 2016), and for supersonic Mach number (Foysi and Friedrich, 2005). Exploiting the similarity between the governing equation of a passive scalar (Eq. (1d)) and the streamwise momentum equation (Eq. (1b)), we introduce a transformation for the mean scalar field which mimics the TL transformation (Eq. (8)), as follows

$\phi_{T}(y, z)=\int_{0}^{y} g_{T}(\eta, z) \frac{\partial \tilde{\phi}}{\partial \eta}(\eta, z) \mathrm{d} \eta$,

with $g_{T}$ given in Eq. (9). Fig. 10 shows the inner-scaled transformed passive scalar profiles, compared with the correlations developed by Kader (1981) for incompressible pipe flow, which include an inertial layer with logarithmic dependence on the wall distance. The figure does in fact show reasonable collapse of the $\phi_{T}$ distributions with respect to $z$ across the different flow cases and with the incompressible fittings. This findings supports our conjecture that the TL transformation can be extended to predict the behavior of passive scalars in compressible flow. We recall that the uniform forcing $\Phi$ in the passive scalar Eq. (1d), equivalent to the uniform pressure gradient in the momentum equation $\Pi$ plays a relevant role for the occurrence of the logarithmic layer. Indeed, the mean temperature, which is controlled by a non-uniform spatial forcing, $\Psi$ in (12), does not exhibit a sizable logarithmic layer, Fig. 7. Again based on the close similarity between $\phi$ and $u$, we consider a generalization of Eq. (13) to relate temperature and passive scalars, namely
$\frac{\tilde{T}}{T_{w}}=1+\frac{T_{r g}-T_{w}}{T_{w}} \frac{\widetilde{\phi}}{\widetilde{\phi}_{e}}+\frac{\widetilde{T}_{e}-T_{r g}}{T_{w}}\left(\frac{\widetilde{\phi}}{\widetilde{\phi}_{e}}\right)^{2}$,

where $\widetilde{\phi}_{e}$ denotes the mean value of the passive scalar at the duct centerline. Fig. 11 shows the scatter plots of temperature as a function of the mean passive scalar for all points in the duct cross section. As for the velocity field, very good collapse of the supersonic DNS data with the predictions of Eq. 15is observed, with the dependence of $\tilde{T}$ on $\tilde{\phi}$ only slightly less universal than its dependence on $\tilde{u}$. We conclude that integration of Eq. (14) can be used to reconstruct the full passive scalar field for assigned values of the bulk Reynolds and Mach numbers.

\section{Instantaneous flow field}

The general flow organization is scrutinized through visualizations of velocity, passive scalar and temperature fluctuations in the wallparallel and cross-stream planes. Fig. 12 shows instantaneous streamwise velocity fluctuations in wall-parallel planes at a distance $y_{T}^{*}=15$. The velocity fluctuations in the buffer layer are organized in alternating low/high velocity streaky structures, elongated in the streamwise direction. The figure shows that the azimuthal spacing of the streaks decreases with the Reynolds number, and in particular we find that the typical spacing is of order $100 \delta_{v}^{*}$, the same observed in incompressible flows, thus further supporting the use of the semi-local scaling defined in (10)-(11) as effective wall units in compressible duct flow. A complementary picture of the flow field is provided by the flow snapshots in cross-stream planes, shown in Fig. 13. Whereas at low Reynolds number only eddies with $O(h)$ size are found, scale separation clearly emerges in flow case D15B, which also includes near-wall small-scale structures eddies in addition to the core eddies. As in incompressible wallbounded flows, the passive scalar field shows substantial correlation with the streamwise velocity field, but it is characterized by sharper interfaces between regions with positive and negative fluctuations, owing to the absence of the pressure gradient term, whose effect is to smoothen the velocity field (Pirozzoli et al., 2016). Temperature fluctuations also show very similar organization as velocity and passive scalar fluctuations throughout the Mach number range under study, despite the previously noted differences between their mean fields. The picture thus emerges that the qualitative structure of turbulence in duct flow is not substantially changed by compressibility, and the essential universal features of turbulent wall-bounded flows are retained.

\section{Conclusions}

We have carried out DNS of developed compressible flow in a square duct at various Mach and Reynolds numbers, with the aim of clarifying the behavior of the mean velocity, temperature and passive scalar fields. We have found that all the flow cases exhibit the same typical secondary flow structure including eight counter-rotating eddies, which act to supply momentum to the duct corners. The cross-stream velocity peaks are found to scale reasonably well with the bulk flow velocity, regardless of Mach and Reynolds number, with maximum value of about $2 \% u_{b}$, which is also consistent with the incompressible case (Pirozzoli et al., 2018). For the range of Mach and Reynolds numbers herein considered we find that the TL compressibility transformation for the mean streamwise velocity developed for plane channel flow (Trettel and Larsson, 2016) also well applies to square ducts, provided the nearest wall is considered in defining the vertical coordinate. The DNS data in fact suggest close similarity of the transformed velocity distributions with those in incompressible pipe flow at matching values of an equivalent friction Reynolds number, defined in Eq. (10).

Regarding the temperature field, we find that the various contributions to its budget equation scale well when expressed in stretched $\left(y_{T}^{*}, z_{T}^{*}\right)$ inner-units, irrespective of the Mach and Reynolds numbers. Similar to the incompressible case, we find that mean convection is mainly relevant at the duct corners, whereas it plays a minor role with 
(a)

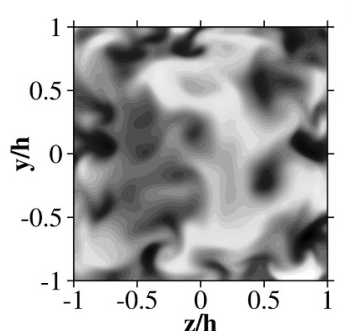

(d)

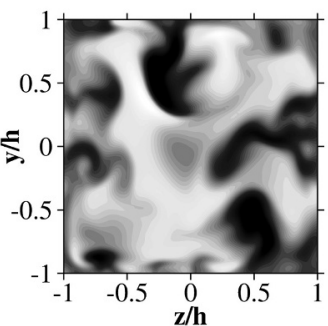

(g)

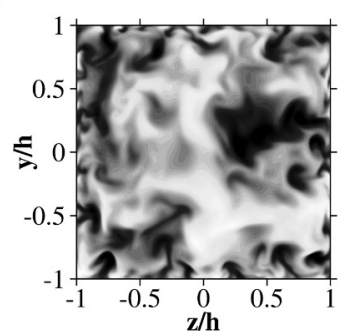

(j)

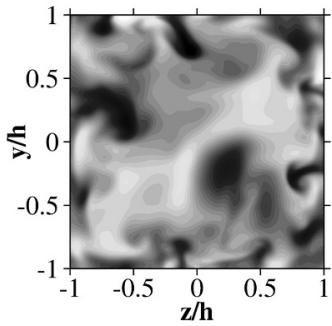

(b)

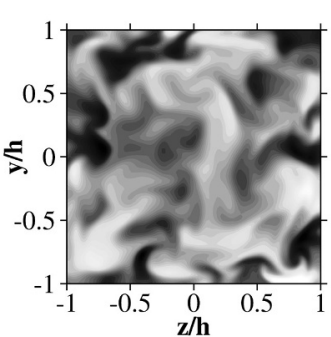

(e)

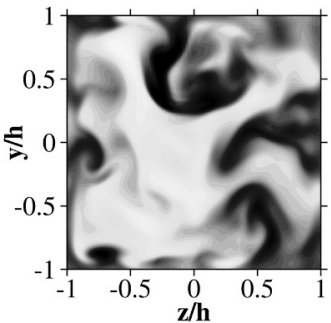

(h)

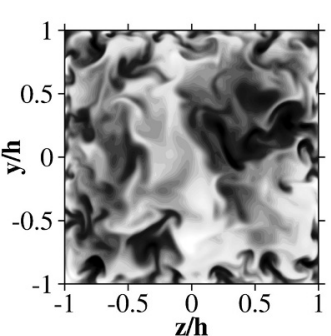

$(\mathrm{k})$

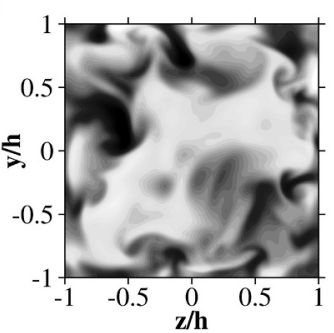

(c)

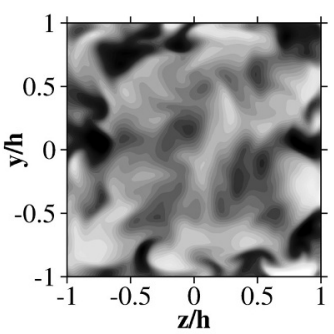

(f)

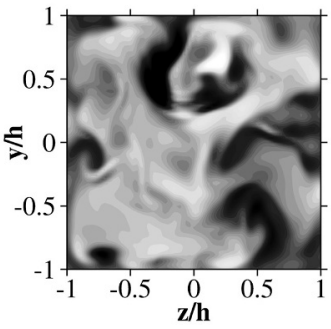

(i)

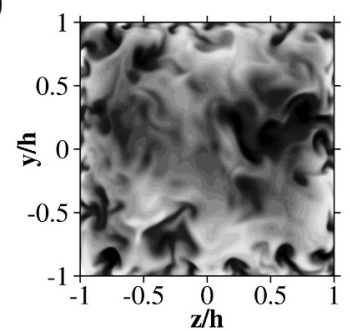

(l)

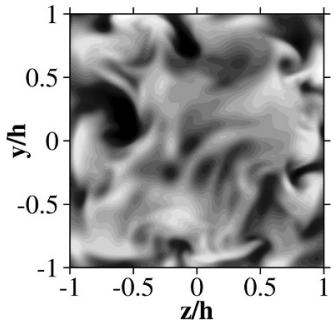

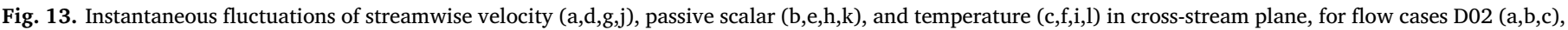

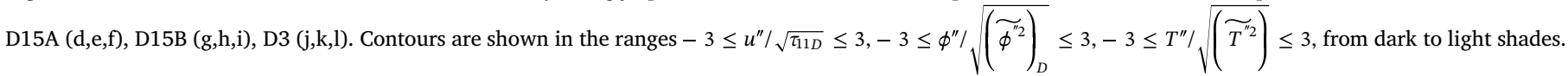

respect to the other terms in the bulk region. Viscous diffusion and dissipation contribute most to the budget, and they are partially balanced by turbulent heat transport in the buffer layer. As a consequence, mean temperature does not exhibit any logarithmic layer, nor universality with respect to Reynolds and Mach number variations. We further show that a generalized form of Waltz' equation can be used to relate the mean velocity and passive scalar fields with the mean temperature field.

Exploiting the formal similarity between the governing equation of a passive scalar with spatially uniform heating and the streamwise momentum equation, we show that the TL transformation can be generalized to achieve universal mean passive scalar profiles, which attain the classical logarithmic form. Differences between passive scalars and the temperature fields are then attributable to the strongly non-uniform distribution of the aerodynamic heating term in the latter case. These results point to a relatively simple representation of the mean flow properties in compressible duct flow, which may be exploited in for the development of improved predictive formulas for friction and heat transfer across the range of Reynolds and Mach numbers, which will be the subject of further study.

Flow statistics are available at the web page http://newton.dima. uniroma1.it/database

\section{Acknowledgments}

We acknowledge that the results reported in this paper have been achieved using the PRACE Research Infrastructure resource MARCONI based at CINECA, Casalecchio di Reno, Italy.

\section{References}

Bradshaw, P., 1987. Turbulent secondary flows. Annu. Rev. Fluid Mech. 19 (1), 53-74. Bradshaw, P., Huang, G., 1995. The law of the wall in turbulent flow. Proc. R. Soc. Lond A 451 (1941), 165-188.

Coleman, G., Kim, J., Moser, R., 1995. A numerical study of turbulent supersonic isothermal-wall channel flow. J. Fluid Mech. 305, 159-183.

Davis, D., Gessner, F., Kerlick, G., 1986. Experimental and numerical investigation of supersonic turbulent flow through a square duct. AIAA J. 24 (9), 1508-1515.

van Driest, E., 1951. Turbulent boundary layer in compressible fluids. J. Aero. Sci. 18, $145-160$.

Duan, L., Beekman, I., Martin, M., 2010. Direct numerical simulation of hypersonic turbulent boundary layers. Part 2. Effect of wall temperature. J. Fluid Mech. 655, 419-445.

El Khoury, J., Schlatter, P., Noorani, A., Fischer, P., Brethouwer, G., Johansson, A., 2013. Direct numerical simulation of turbulent pipe flow at moderately high Reynolds numbers. Flow Turbul. Combust. 91 (3), 475-495.

Foysi, H., Friedrich, R., 2005. DNS of passive scalar transport in turbulent supersonic channel flow. High Performance Computing in Science and Engineering, Munich 2004. Springer, pp. 107-117. 
Ghosh, S., Foysi, H., Friedrich, R., 2010. Compressible turbulent channel and pipe flow: similarities and differences. J. Fluid Mech. 648, 155-181.

Ghosh, S., Sesterhenn, J., Friedrich, R., 2008. Large-eddy simulation of supersonic turbulent flow in axisymmetric nozzles and diffusers. Int. J. Heat Fluid Flow 29 (3), 579-590.

Huang, P., Coleman, G., Bradshaw, P., 1995. Compressible turbulent channel flows: DNS results and modeling. J. Fluid Mech. 305, 185-218.

Kader, B., 1981. Temperature and concentration profiles in fully turbulent boundary layers. Int. J. Heat Mass Transf. 24 (9), 1541-1544.

Klein, M., Sadiki, A., Janicka, J., 2003. A digital filter based generation of inflow data for spatially developing direct numerical or large eddy simulations. J. Comput. Phys. 186 (2), 652-665.

Mani, M., Babcock, D., Winkler, C., Spalart, P., 2013. Predictions of a supersonic turbulent flow in a square duct. AIAA Paper 860, 2013.

Modesti, D., 2017. Direct numerical simulation of internal compressible flows at high Reynolds number: numerical and physical insight. La Sapienza Unversità di Roma. Published online: http://hdl.handle.net/11573/939526

Modesti, D., Pirozzoli, S., 2016. Reynolds and Mach number effects in compressible turbulent channel flow. Int. J. Heat Fluid Flow 59, 33-49.

Modesti, D., Pirozzoli, S., 2018. An efficient semi-implicit solver for direct numerical simulation of compressible flows at all speeds. J. Sci. Comput. 75 (1), 308-331.

Modesti, D., Pirozzoli, S., Orlandi, P., Grasso, F., 2018. On the role of secondary motions in turbulent square duct flow. J. Fluid Mech. 847.

Morajkar, R., Gamba, M., 2016. Turbulence characteristics of supersonic corner flows in a low aspect ratio rectangular channel. 54th AIAA Aerospace Sciences Meeting. pp. 1590.

Morkovin, M., 1962. Effects of compressibility on turbulent flows. Mécanique de la Turbulence. A. Favre, pp. 367-380.
Nikuradse, J., 1926. Untersuchung über die Geschwindigkeitsverteilung in turbulenten Strömungen. Vdi-verlag.

Pinelli, A., Uhlmann, M., Sekimoto, A., Kawahara, G., 2010. Reynolds number dependence of mean flow structure in square duct turbulence. J. Fluid Mech. 644, 107-122.

Pirozzoli, S., 2010. Generalized conservative approximations of split convective derivative operators. J. Comput Phys. 229 (19), 7180-7190.

Pirozzoli, S., Bernardini, M., Orlandi, P., 2016. Passive scalars in turbulent channel flow at high Reynolds number. J. Fluid Mech. 788, 614-639.

Pirozzoli, S., Modesti, D., Orlandi, P., Grasso, F., 2018. Turbulence and secondary motions in square duct flow. J. Fluid Mech. 840, 631-655.

Prandtl, L., 1927. Über den reibungswiderstand strömender luft. Reports of the Aerod. Versuchsanst, Göttingen, Germany, 3rd Series.

Smits, A., Dussauge, J.-P., 2006. Turbulent Shear Layers in Supersonic Flow, Second ed. American Institute of Physics, New York.

Trettel, A., Larsson, J., 2016. Mean velocity scaling for compressible wall turbulence with heat transfer. Phys. Fluids (1994-present) 28 (2), 026102.

Vane, Z., Lele, S., 2015. Prediction of turbulent secondary flows in ducts using equilibrium wall-modeled LES. 53rd AIAA Aerospace Sciences Meeting. pp. 1274.

Vázquez, M., Métais, O., 2002. Large-eddy simulation of the turbulent flow through a heated square duct. J. Fluid Mech. 453, 201-238.

Vinuesa, R., Prus, C., Schlatter, P., Nagib, H., 2016. Convergence of numerical simulations of turbulent wall-bounded flows and mean cross-flow structure of rectangular ducts. Meccanica 51 (12), 3025-3042.

Walz, A., 1959. Compressible turbulent boundary layers with heat transfer and pressure gradient in flow direction. J. Res. Natl. Bur. Stand. 63.

Zhang, Y., Bi, W., Hussain, F., She, Z., 2014. A generalized Reynolds analogy for compressible wall-bounded turbulent flows. J. Fluid Mech. 739, 392-420. 